The Nyström method can produce ill-conditioned systems of linear equations and inaccurate results when applied to integral equations on domains with corners. This defect can already be seen in the simple case of the integral equations arising from the Neumann problem for Laplace's equation. We explain the origin of this instability and show that a straightforward modification to the Nyström scheme, which renders it mathematically equivalent to Galerkin discretization, corrects the difficulty without incurring the computational penalty associated with Galerkin methods. We also present the results of numerical experiments showing that highly accurate solutions of integral equations on domains with corners can be obtained, irrespective of whether their solutions exhibit bounded or unbounded singularities, assuming that proper discretizations are used.

\title{
On the Nyström discretization of integral equations on planar curves with corners
}

\author{
James Bremer ${ }^{\dagger}$ \\ Technical Report YALEU/DCS/TR-1433 \\ July 22, 2010
}

This research was supported in part by the Office of Naval Research, under contract N00014-09-10318 .

$\dagger$ Dept. of Mathematics, Univ. of California at Davis, Davis, CA 95616

Approved for public release: distribution is unlimited.

Keywords: Fast multipole methods, boundary integral equations, corner singularities. 


\title{
ON THE NYSTRÖM DISCRETIZATION OF INTEGRAL EQUATIONS ON PLANAR CURVES WITH CORNERS
}

\author{
JAMES BREMER
}

\begin{abstract}
The Nyström method can produce ill-conditioned systems of linear equations and inaccurate results when applied to integral equations on domains with corners. This defect can already be seen in the simple case of the integral equations arising from the Neumann problem for Laplace's equation. We explain the origin of this instability and show that a straightforward modification to the Nyström scheme, which renders it mathematically equivalent to Galerkin discretization, corrects the difficulty without incurring the computational penalty associated with Galerkin methods. We also present the results of numerical experiments showing that highly accurate solutions of integral equations on domains with corners can be obtained, irrespective of whether their solutions exhibit bounded or unbounded singularities, assuming that proper discretizations are used.
\end{abstract}

\section{INTRODUCTION}

It is well known that Neumann boundary value problems for Laplace's equation can be formulated as second kind integral equations. In the case of a compact simply-connected planar domain $\Omega$ with Lipschitz boundary $\partial \Omega$, the exterior problem

$$
\begin{array}{ll}
\Delta u=0 & \text { in } \Omega^{c} \\
\frac{\partial u}{\partial \nu}=g & \text { on } \partial \Omega
\end{array}
$$

is solvable when $g$ is in the space $L_{0}^{2}(\partial \Omega)$ of square integrable functions of zero mean on $\partial \Omega$. Here, $\nu$ is the outward-pointing unit normal of $\partial \Omega$ and the boundary condition must be understood as a limit in the appropriate non-tangential sense. If the additional condition

$$
u(x)=O\left(\frac{1}{|x|}\right) \quad \text { as }|x| \rightarrow \infty
$$

is imposed, then the solution is unique. The usual integral equation method exploits the observation that the solution $u$ can be represented uniquely in the form of a single-layer charge distribution $\sigma$ on $\partial \Omega$; that is, as

$$
u(x)=\frac{1}{2 \pi} \int_{\partial \Omega} \log |x-y| \sigma(y) d s(y) .
$$

The charge distribution $\sigma$ which, when inserted into (1.3), produces the solution of (1.1) can be determined by solving the integral equation

$$
\sigma(x)+N \sigma(x)=2 g(x), \quad x \in \partial \Omega,
$$

where $N$ is the operator

$$
N \sigma(x)=\frac{1}{\pi} \int_{\partial \Omega}\left(\frac{\partial}{\partial \nu_{x}} \log |x-y|\right) \sigma(y) d s(y) .
$$

In this article, we will consider domains $\partial \Omega$ which are piecewise smooth with corner points. By corner point, we mean a point on the boundary curve $\partial \Omega$ at which the limits of the normal derivative

Date: July 22, 2010. 
as one approaches from the clockwise and the counter-clockwise directions exist but are not equal. For these domains, the kernel

$$
K(x, y)=\frac{1}{\pi} \frac{\partial}{\partial \nu_{x}} \log |x-y|
$$

of the integral operator $N$ is smooth except on the set consisting of points $(x, y) \in \partial \Omega \times \partial \Omega$ such that either $x$ or $y$ is a corner point.

The Nyström method is one of the standard approaches to the discretization of the integral equation (1.4). It proceeds by fixing an appropriate quadrature formula $x_{1}, \ldots, x_{n}, w_{1}, \ldots, w_{n}$ and approximating the values of the charge distribution $\sigma$ at the quadrature nodes $x_{1}, \ldots, x_{n}$ by solving the system

$$
\sigma\left(x_{i}\right)+\sum_{j=1}^{n} K\left(x_{i}, x_{j}\right) \sigma\left(x_{j}\right) w_{j}=2 g\left(x_{i}\right), \quad i=1, \ldots, n,
$$

of $n$ linear equations in the $n$ unknowns $\sigma\left(x_{1}\right), \ldots, \sigma\left(x_{n}\right)$.

For many domains, the linear system (1.7) is an effective means for solving the integral equation (1.4). If the boundary of $\partial \Omega$ is twice continuously differentiable, then $N$ is compact as an operator on the Banach space $C(\partial \Omega)$ of continuous functions on $\partial \Omega$ endowed with the uniform norm. The boundedness and invertibility of $I+N$ as an operator on $C(\partial \Omega)$ follow from the Fredholm theory. Moreover, bounds on the operator norms of $I+N$ and $(I+N)^{-1}$ can be used to estimate the $l^{\infty}$ condition number of the linear system (1.7). Convergence results follow from similar considerations; for instance, if the domain $\Omega$ is smooth, then exponential convergence can be achieved by choosing an appropriate quadrature formula. See, for instance, [8] for a discussion of the classical Riesz theory and its application to the numerical solution of the integral equations of potential theory.

When the boundary $\partial \Omega$ is merely Lipschitz, this analysis breaks down. In this case, $I+N$ is bounded and invertible as an operator

$$
I+N: L_{0}^{2}(\partial \Omega) \rightarrow L_{0}^{2}(\partial \Omega)
$$

see, for instance, [5]. But now $(I+N)^{-1}$ is not bounded with respect to the uniform norm; indeed, there are functions $g \in C(\partial \Omega)$ such that the charge distribution $\sigma$ satisfying the integral equation (1.4) is unbounded (see Section 4.3). So the usual estimates on the $l^{\infty}$ condition number of the linear system (1.7) no longer apply. This difficulty led to conditioning problems in [3] and it appears to be the motivation for [4], which notes that the Nyström method, when applied to boundary integral operators on domains with corners, can produce ill-conditioned linear systems and inaccurate results.

Stability can be restored by discretizing $I+N$ as an operator on $L_{0}^{2}(\partial \Omega)$ rather than $C(\partial \Omega)$. This can be accomplished by switching from Nyström to Galerkin discretization. That is, by choosing an appropriate orthonormal basis $\psi_{1}, \ldots, \psi_{n}$ and discretizing the integral operator $I+N$ via the $n \times n$ matrix $A$ with entries

$$
A_{i j}=\delta_{i j}+\int_{\partial \Omega} \int_{\partial \Omega} K(x, y) \psi_{j}(y) \psi_{i}(x) d s(y) d s(x) .
$$

Bounds for the condition number of $A$ as a function of singular values of the operator

$$
I+N: L_{0}^{2}(\partial \Omega) \rightarrow L_{0}^{2}(\partial \Omega)
$$

can be readily obtained. There is, however, a disadvantage to this approach: Galerkin discretizations are computationally more expensive and more complicated to construct than Nyström discretizations because of the need to evaluate double integrals numerically.

It is the purpose of this article to show that a second remedy, which does not suffer from this disadvantage, is available. In particular, the Nyström scheme can be modified to produce discretizations which reflect the properties of $I+N$ as an operator on $L_{0}^{2}(\partial \Omega)$. The modified scheme results in well-conditioned linear systems approximating (1.4) on domains with corners whose solutions are highly accurate. Moreover, this method is simpler and more efficient than Galerkin discretization in that it does not require the numerical evaluation of double integrals. 
There are further difficulties complicating the discretization of integral equations on domains with corners: the operator $N$ is not compact and both the kernel $K(x, y)$ and solutions of the equation (1.4) are singular. It has long been standard practice to address the first problem by forming a modified boundary curve $\partial \Omega_{\delta}$ which excludes from $\partial \Omega$ a $\delta$-neighborhood of each corner point, introducing the compact integral operator

$$
N_{\delta}(x)=\frac{1}{\pi} \int_{\partial \Omega_{\delta}}\left(\frac{\partial}{\partial \nu_{x}} \log |x-y|\right) \sigma(y) d s(y)
$$

and considering, in lieu of (1.4), the integral equation

$$
I+N_{\delta}=2 g(x), \quad x \in \partial \Omega_{\delta} .
$$

It is well established that solutions $\sigma_{\delta}$ of (1.9) converge to those of (1.4) in a distributional sense; that is,

$$
\int_{\partial \Omega_{\delta}} \log |x-y| \sigma_{\delta}(y) d s(y) \rightarrow \int_{\partial \Omega} \log |x-y| \sigma(y) d s(y) \text { as } \delta \rightarrow 0
$$

for $x \in \Omega^{c}$. To combat the difficulties associated with singular solutions and kernels, the Nyström discretization of (1.9) is usually formed using graded mesh quadratures; that is, with the help of quadratures of various types which become increasingly dense near corner points.

Moreover, while the discretization of boundary integral equations on domains with corners via the Nyström method and graded mesh quadrature can be made accurate and stable, this is not by itself a reasonable approach to the solution of boundary integral equations on large-scale domains with corners. The systems of linear equations arising from such domains are excessively large and must be compressed in some fashion. In Section 3, we present a scheme for the compression of linear systems obtained by discretizing boundary integral equations on domains with corners which is based on ideas introduced in [2] and [3]. In conjunction with fast multipole methods and fast direct solvers, it allows for the very rapid solution of boundary integral equations on large-scale domains with corners. Although we only consider the Neumann problem for Laplace's equation here, the scheme of Section 3 applies to a wide class of boundary value problems; see, for instance, [3] where a similar approach is used to solve the Dirichlet problem for the Helmholtz equation at low wavenumbers. Moreover, certain components of these schemes generalize readily to the threedimensional environment. The specific approach discussed in Section 3 can be readily extended to this setting, for instance.

This article is structured as follows. In Section 2, we discuss the Nyström discretization of integral operators on domains with corners. Section 3 gives the details of a scheme for the compression of systems of linear equations arising from boundary integral equations on domains with corners. In Section 4, we present the results of numerical experiments showing that the boundary integral equation (1.4) can be solved stably and to high accuracy with graded mesh quadrature provided appropriate discretizations are used. Finally, we close with a few remarks on the numerical solution of integral equations on domains with corners in Section 5.

\section{Discretization of integral Equations on DOMAins With CORNERS}

2.1. Discretization of compact operators acting on $L^{2}$ spaces. If $(X, \mu)$ is a measure space, then we say that a mapping $\Phi$ of $M \subset L^{2}(X, d \mu)$ into $\mathbb{C}^{n}$ preserves inner products provided

$$
\Phi(f) \cdot \Phi(g)=\int_{X} f(x) g(x) d \mu(x)
$$

for all $f, g \in M$.

Consider a compact operator $S: L^{2}(X, d \mu) \rightarrow L^{2}(X, d \mu)$. It is well known that $S$ admits singular value decomposition. We will denote by $\left\{\lambda_{j}\right\}$ the singular values of $S$ and for each $j$, we will let $u_{j}$ and $v_{j}$ be the left and right singular functions corresponding to $\lambda_{j}$. In the event that the rank of $S$ is finite, we set $\lambda_{j}=0$ and $u_{j}=v_{j}=0$ for all $j$ greater than the rank of $S$. Moreover, we will let $U_{\epsilon}$ and $V_{\epsilon}$ be the subspaces spanned by $\left\{u_{j} \mid \lambda_{j}<\epsilon\right\}$ and $\left\{v_{j} \mid \lambda_{j}<\epsilon\right\}$, respectively. 
We say a matrix $A$ in $\mathbb{C}^{n \times n}$ is an inner product preserving discretization of $S$ of accuracy $\epsilon$ if there exist inner product preserving mappings $\Phi$ of $U_{\epsilon}$ into $\mathbb{C}^{n}$ and $\Psi$ of $V_{\epsilon}$ into $\mathbb{C}^{n}$ such that the diagram

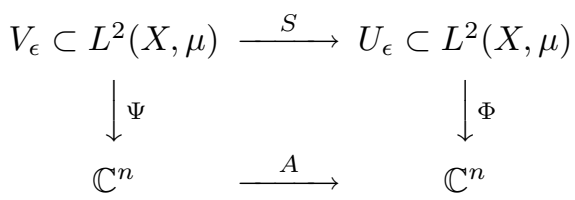

commutes. This is equivalent to requiring that

$$
A \Psi(f) \cdot \Phi(g)=\int_{X} S f(x) g(x) d \mu(x)
$$

for all $f \in V_{\epsilon}$ and $g \in U_{\epsilon}$. One of the key properties of such a discretization is that

$$
A^{*} A \Psi(f) \cdot \Psi(g)=A \Psi(f) \cdot A \Psi(g)=\int_{X} S f(x) S g(x) d \mu(x)=\int_{X} S^{*} S f(x) g(x) d \mu(x)
$$

for all $f, g \in V_{\epsilon}$, from which it follows that the singular values of the restriction of the matrix $A$ to $\Psi\left(V_{\epsilon}\right)$ are those of the restriction of the operator $S$ to $V_{\epsilon}$.

Remark 2.1. Galerkin discretization is perhaps the easiest way to produce an inner product preserving discretization of a compact operator $S: L^{2}(X, d \mu) \rightarrow L^{2}(X, d \mu)$. Let $f_{1}, \ldots, f_{n}$ be an orthonormal basis spanning $V_{\epsilon}$ and let $g_{1}, \ldots, g_{n}$ be an orthonormal basis spanning $U_{\epsilon}$. The mapping which takes functions in $V_{\epsilon}$ to their coefficient expansions with respect to the basis $\left\{f_{j}\right\}$ is inner product preserving, as is the mapping which takes functions in $U_{\epsilon}$ to their coefficient expansions in the basis $\left\{g_{i}\right\}$. The Galerkin discretization of $S$ with respect to these bases, which is the $n \times n$ matrix $A$ with entries

$$
A_{i j}=\int_{X} S f_{i}(x) g_{j}(x) d \mu(x)
$$

is then an inner product preserving discretization of $S$ of accuracy $\epsilon$.

2.2. Inner product preserving Nyström discretizations. Now suppose that $S$ is a compact integral operator $L^{2}(X, d \mu) \rightarrow L^{2}(X, d \mu)$ whose kernel $L(x, y)$ is defined pointwise everywhere and let $V_{\epsilon}$ and $U_{\epsilon}$ be as in the preceding section. A quadrature rule of the form

$$
\int_{X} f(y) d \mu(y) \approx \sum_{l=1}^{n} f\left(x_{l}\right) w_{l}
$$

with positive weights induces a mapping $\Phi$ of $L^{2}(X, d \mu)$ into $\mathbb{C}^{n}$; to wit,

$$
\Phi(f)=\left(\begin{array}{c}
f\left(x_{1}\right) \sqrt{w_{1}} \\
f\left(x_{2}\right) \sqrt{w_{2}} \\
\vdots \\
f\left(x_{n}\right) \sqrt{w_{n}}
\end{array}\right) .
$$

If the quadrature rule (2.1) is exact for products of the form $f g$ with $f, g \in V_{\epsilon}$ and $h f$ with $h, f \in U_{\epsilon}$, then $\Phi$ is an inner product preserving mapping of $U_{\epsilon}$ and $V_{\epsilon}$ into $\mathbb{C}^{n}$.

Moreover, the choice of quadrature $x_{1}, \ldots, x_{n}, w_{1}, \ldots, w_{n}$ induces a mapping of the space of integrals operators on $L^{2}(X, d \mu)$ with pointwise defined kernels into the space of operators on $\mathbb{C}^{n}$. In particular, $S$ is mapped to the $n \times n$ matrix $A$ with entries

$$
A_{i j}=L\left(x_{i}, x_{j}\right) \sqrt{w_{i}} \sqrt{w_{j}} .
$$

If the quadrature rule is sufficiently dense to integrate the products of the kernel with the functions in $U_{\epsilon}$ and $V_{\epsilon}$, then we will have

$$
A \Phi(f) \cdot \Phi g(x)=\int_{X} S f(x) g(x) d s(x)
$$

for $f \in V_{\epsilon}$ and $g \in U_{\epsilon}$; that is, $A$ will be an inner product preserving discretization of the integral operator $S$ of accuracy $\epsilon$. 
This notion of discretization, when applied to the operator $I+N$, leads to the $n \times n$ matrix $B$ with entries

$$
B_{i j}=\delta_{i j}+K\left(x_{i}, x_{j}\right) \sqrt{w_{i}} \sqrt{w_{j}},
$$

where $K(x, y)$ is the kernel (1.6) of $N$. This is in contrast to the standard Nyström discretization of $I+N$, which is the matrix $C$ with entries

$$
C_{i j}=\delta_{i j}+K\left(x_{i}, x_{j}\right) w_{j}
$$

The salient difference between the matrices $B$ and $C$ is that the singular values of $B$ approximate those of $I+N$ while the singular values of $C$ do not. Since the operator $I+N$ and its inverse have well-behaved $L^{2}(\partial \Omega)$ operator norms on most curves of interest, it follows that the matrix $B$ is well-conditioned in typical cases, including on many singular domains. The matrix $C$, on the other hand, is generally ill-conditioned when $\partial \Omega$ is singular.

The solution of the integral equation (1.4) using the inner product preserving discretization $B$ proceeds by letting $y$ be the vector of length $n$ with entries

$$
y_{i}=g\left(x_{i}\right) \sqrt{w_{i}}
$$

and inverting the linear system $B z=y$. The entries of the resulting vector $z$ are the values of the charge distribution $\sigma$ satisfying (1.4) at the quadrature nodes $x_{i}$ scaled by the square roots of the corresponding quadrature weights; that is,

$$
z_{i}=\sigma\left(x_{i}\right) \sqrt{w_{i}} .
$$

In other words, the analog of (1.7) is the system

$$
\sigma\left(x_{i}\right) \sqrt{w_{i}}+\sum_{j=1}^{n} K\left(x_{i}, x_{j}\right) \sqrt{w_{i}} \sqrt{w_{j}} \sigma\left(x_{j}\right)=2 g\left(x_{i}\right) \sqrt{w_{i}}, \quad i=1, \ldots, n,
$$

of $n$ linear equations in the $n$ unknowns

$$
\sigma\left(x_{1}\right) \sqrt{w_{1}}, \sigma\left(x_{2}\right) \sqrt{w_{2}}, \ldots, \sigma\left(x_{n}\right) \sqrt{w_{n}} .
$$

We will refer to linear systems of this type as inner product preserving discretizations of the integral equation (1.4). Note that the vector obtained from solving the linear system (2.4) is the image of the charge distribution $\sigma$ under the inner product preserving mapping $\Phi$; i.e., we solve for $\sigma$ in an $L^{2}(\partial \Omega)$ sense rather than in a pointwise sense.

Remark 2.2. We have neglected certain details in this section in order to simply the discussion. Specifically, in finite precision arithmetic it is inevitable that the quadrature formula (2.1) will not hold exactly for the requisite integrands. This means that our discretizations will suffer from inaccuracies both as a result of finite rank approximation and from quadrature error. Limitations of this type, however, apply to all methods for the discretization of integral operators.

Remark 2.3. Inner product preserving Nyström discretizations can be produced in the case of integral operators whose kernels are not defined pointwise provided the appropriate interpolatory quadratures are available.

\section{Compression of LineAR SYSTEMS ARISING FROM INTEGRAL EQUATIONS ON DOMAINS With CORNERS}

We now describe a mechanism for the compression of systems of linear equations arising from boundary integral equations on domains with corners. It is a variant of the schemes of [3] and [2] which operates via two tools, generalized quadrature and charge bases. Generalized quadrature and charge bases are discussed in Sections 3.1 and 3.2, respectively, and the compression scheme proper is detailed in Section 3.3. 
3.1. Generalized quadrature. We define the $\epsilon$-rank of an $n \times m$ matrix $A$ to be the least integer $k$ such that the $k$ largest singular values of $A$ are greater than $\epsilon$, assuming that such an integer exists. If it does not, then we say that the $\epsilon$-rank of $A$ is $\min (m, n)$.

If $f_{1}, \ldots, f_{m}$ is a collection of functions in $L^{2}(X, \mu)$ and $\Phi$ is an inner product preserving mapping of the span of the $f_{j}$ into $\mathbb{C}^{n}$, then we define the $\epsilon$-rank of $f_{1}, \ldots, f_{m}$ to be the $\epsilon$-rank of the $n \times m$ matrix

$$
A=\left(\begin{array}{lll}
\Phi\left(f_{1}\right) & \ldots & \Phi\left(f_{m}\right)
\end{array}\right)
$$

whose columns consist of the images of the $f_{j}$ under the embedding $\Phi$.

As described in the last section, a quadrature formula $x_{1}, \ldots, x_{n}, w_{1}, \ldots, w_{n}$ with positive weights which integrates products of the functions $f_{1}, \ldots, f_{m}$ induces an inner product preserving mapping of the span of the $f_{j}$ into $\mathbb{C}^{n}$. The following theorem on the existence of efficient quadrature formulas is an almost immediate consequence of that fact.

THEOREM 3.1. If $x_{1}, \ldots, x_{n}, w_{1}, \ldots, w_{n}$ is a quadrature rule with positive weights which integrates products of the functions $f_{1}, \ldots, f_{m} \in L^{2}(X, d \mu)$, then for every $\epsilon>0$ there exists a quadrature rule $y_{1}, \ldots, y_{k}, v_{1}, \ldots, v_{k}$, whose length $k$ is the $\epsilon$-rank of the collection $f_{1}, \ldots, f_{m}$, such that

$$
\sum_{i=1}^{m}\left|\int_{X} f(x) d \mu(x)-\sum_{j=1}^{k} f\left(y_{j}\right) v_{j}\right|^{2}<(1+m k(m-k)) \epsilon^{2} .
$$

Quadratures of this type can be constructed by finding a sparse least squares solution to a system of linear equations. Let

$$
F=\left(\begin{array}{cccc}
f_{1}\left(x_{1}\right) \sqrt{w_{1}} & f_{1}\left(x_{2}\right) \sqrt{w_{2}} & \ldots & f_{1}\left(x_{n}\right) \sqrt{w_{n}} \\
f_{2}\left(x_{1}\right) \sqrt{w_{1}} & f_{2}\left(x_{2}\right) \sqrt{w_{2}} & \ldots & f_{2}\left(x_{n}\right) \sqrt{w_{n}} \\
\vdots & & \vdots & \\
f_{m}\left(x_{1}\right) \sqrt{w_{1}} & f_{m}\left(x_{2}\right) \sqrt{w_{2}} & \ldots & f_{m}\left(x_{n}\right) \sqrt{w_{n}}
\end{array}\right)
$$

and

where

$$
b=\left(\begin{array}{c}
b_{1} \\
b_{2} \\
\vdots \\
b_{m}
\end{array}\right)
$$

$$
b_{j}=\int_{X} f_{j}(x) d \mu(x), \quad j=1, \ldots, m .
$$

If the $\epsilon$-rank of the collection $f_{1}, \ldots, f_{m}$ is $k$, then a vector $z \in \mathbb{C}^{n}$ with no more than $k$ nonzero entries such that

$$
\|F z-b\|_{2}<\epsilon \sqrt{1+m k(m-k)}
$$

can be constructed stably in $O\left(n m^{2}\right)$ floating-point operations (see [6]). If we let $i_{1}, \ldots, i_{k}$ denote the indices of the nonzero entries of the vector $z$ and set

$$
y_{j}=x_{i_{j}}, \quad j=1, \ldots, k,
$$

and

$$
v_{j}=z_{i_{j}} \sqrt{w_{i_{j}}}, \quad j=1, \ldots, k,
$$

then $y_{1}, \ldots, y_{k}, v_{1}, \ldots, v_{k}$ is a $k$-point quadrature for the functions $f_{1}, \ldots, f_{m}$. See [1] for an extended discussion of the construction of such quadratures.

Remark 3.1. Note the scaling by square roots of quadrature weights in (3.1). As in the case of integral operators, the use of inner product preserving embeddings of this type leads to bounds on the condition number of the matrix (3.1). Their omission, by contrast, generally results in illconditioning. For instance, if the functions $f_{j}$ are taken to be the monomials $x^{j}$ on the interval $[-1,1]$ and square roots are omitted, then (3.1) becomes a Vandermonde matrix. 
3.2. Charge bases. In this section, we show that a basis spanning the space of restrictions of solutions of the integral equation (1.4) to a corner region can be constructed under a mild assumption on the right-hand sides of (1.4). We will refer to a basis of this type as a charge basis for the corner region.

Let $\Gamma_{0}$ be a corner region which is part of the boundary curve $\partial \Omega$ and let $B$ be the disc of minimum radius centered at the corner point of $\Gamma_{0}$ which contains $\Gamma_{0}$. Denote by $\Gamma_{1}$ the portion of the contour $\partial \Omega$ contained in $2 B \backslash B$, and by $\Gamma_{2}$ the portion of $\partial \Omega$ contained in the complement of the disc $2 B$. Figure 1 depicts the situation. Our assumption on right-hand side $g(x)$ of $(1.4)$ is that it is the normal derivative of a function satisfying the Laplace equation in the disc $2 B$.

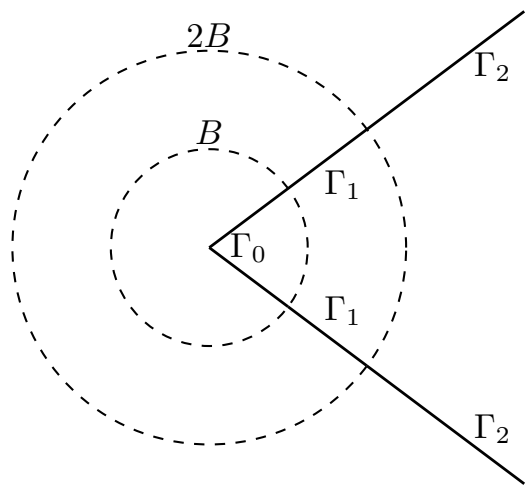

Figure 1: The geometry assumed for the charge basis construction.

The boundary integral equation

$$
\sigma(x)+\int_{\partial \Omega} K(x, y) \sigma(y) d s(y)=2 g(x), \quad x \in \partial \Omega,
$$

can be rearranged as

$$
\sigma(x)+\int_{\Gamma_{0}} K(x, y) \sigma(y) d s(y)=2 g(x)-\int_{\Gamma_{2}} K(x, y) \sigma(y) d s(y)-\int_{\Gamma_{1}} K(x, y) \sigma(y) d s(y), \quad x \in \partial \Omega .
$$

The charge basis construction procedure operates by considering the restricted integral equation

$$
\sigma(x)+\int_{\Gamma_{0}} K(x, y) \sigma(y) d s(y)=2 g(x)-\int_{\Gamma_{2}} K(x, y) \sigma(y) d s(y)-\int_{\Gamma_{1}} K(x, y) \sigma(y) d s(y), \quad x \in \Gamma_{0},
$$

and introducing series approximations for the terms on the right-hand side. By solving this restricted equation for each term of each approximation, we form a set of functions which spans the space of solutions of equation (3.4). Of course, the restriction of any solution of the integral equation (3.2) to $\Gamma_{0}$ satisfies (3.4), so such a collection of functions is a charge basis.

The first approximation depends on our assumption on the right-hand side, namely, that $g$ is the restriction to $\partial \Omega$ of the normal derivative of a function $u$ which is harmonic in the disc $2 B$. It is well known that $u$ can be represented as a multipole expansion

$$
u(x)=\sum_{j=0}^{\infty} \alpha_{j} r^{j} \cos (j \theta)+\beta_{j} r^{j} \sin (j \theta)
$$

in the disc $2 B$. Here, $(r, \theta)$ denotes the usual polar coordinate system with respect to the center of the disc $B$. For points $x$ in $B$, this expansion achieves exponential convergence; in particular, there is a constant $C$ such that for $x \in B$ we have

$$
\left|u(x)-\sum_{j=0}^{N} \alpha_{j} r^{j} \cos (j \theta)+\beta_{j} r^{j} \sin (j \theta)\right| \leq C 2^{-N} .
$$


It follows that $g$ can be approximated on $\Gamma_{0}$ as the normal derivative of a finite sum of multipoles; that is, as

$$
\sum_{j=1}^{N_{1}} \alpha_{j} \frac{\partial}{\partial \nu}\left(r^{j} \cos (j \theta)\right)+\beta_{j} \frac{\partial}{\partial \nu}\left(r^{j} \sin (j \theta)\right),
$$

where the number of terms $N_{1}$ grows logarithmically with the precision of the approximation.

Similar considerations apply to the second term in equation (3.4). Since $K(x, y)$ is harmonic and $\Gamma_{0}$ is separated by a distance equal to its radius from $\Gamma_{2}$, for $x$ in $\Gamma_{0}$ we can introduce the approximation

$$
\int_{\Gamma_{2}} K(x, y) \sigma(y) d y \approx \sum_{j=0}^{N_{2}}\left(\gamma_{j} r^{j} \cos (j \theta)+\eta_{j} r^{j} \sin (j \theta)\right),
$$

where once again $(r, \theta)$ is the polar coordinate system with respect to the center of the disc $B$. The number of terms $N_{2}$ in (3.5) also grows as $O\left(\log _{2}(\epsilon)\right)$ with the desired precision $\epsilon$.

The approximation of the last term in (3.4) requires a different strategy since $\Gamma_{0}$ is not separated from $\Gamma_{1}$. Here we exploit the fact that the integral operator $L^{2}\left(\Gamma_{1}\right) \rightarrow L^{2}\left(\Gamma_{0}\right)$ defined by

$$
R \sigma(x)=\int_{\Gamma_{1}} K(x, y) \sigma(y) d s(y)
$$

is compact and, barring complex geometry, of low rank. This means that the operator $R$ can be approximated via a quadrature formula

$$
\int_{\Gamma_{1}} K(x, y) \sigma(y) d s(y) \approx \sum_{j=1}^{N_{3}} K\left(x, y_{j}\right) \sigma\left(y_{j}\right) w_{j} .
$$

The number of terms in (3.7) depends on the desired accuracy of the approximation and the decay of the singular values of integral operator $R$. In most cases of interest, $N_{3}$ grows as $O\left(\log _{3}^{2}(\epsilon)\right)$, where $\epsilon$ is the accuracy achieved; see [3] for a detailed estimate.

It follows that a charge basis can be formed by solving the restricted integral equation (3.4) for the normal derivatives

$$
\frac{\partial}{\partial \nu} r^{j} \cos (j \theta) \text { and } \frac{\partial}{\partial \nu} r^{j} \sin (j \theta), \quad j=1, \ldots, N_{1},
$$

the multipoles

$$
r^{j} \cos (j \theta) \quad \text { and } \quad r^{j} \sin (j \theta), \quad j=0, \ldots, N_{2},
$$

and the kernel functions

$$
K\left(x, y_{j}\right), \quad y_{1}, \ldots, y_{N_{3}} \in \Gamma_{1} .
$$

It is easy to see from our earlier estimates that, in typical cases, the dimension of the resulting basis is $O\left(\log _{3}^{2}(\epsilon)\right)$, where $\epsilon$ is the desired precision for the basis.

3.3. The compression procedure. The mechanisms discussed in the two preceding sections, charge bases and generalized quadrature, can be used to compress the systems of linear equations resulting from boundary integral equations on domains with corners. We now describe one algorithm for doing so; it is a variant of that introduced in [2] and amounts to a local direct solver for corner regions.

\section{Setup for the procedure}

We will consider the discretization of the integral equation (1.4) on a contour $\partial \Omega$ which is piecewise smooth with a corner region $\Gamma_{0}$. By corner region we mean a compact connected subset of $\partial \Omega$ which contains a corner point in its interior. Let $r:[-\delta, \delta] \rightarrow \Gamma_{0}$ be a parameterization of the corner region $\Gamma_{0}$. Also, let $x_{1}, \ldots, x_{n}, w_{1}, \ldots, w_{n}$ be a quadrature rule for functions on the corner region $\Gamma_{0}$ and 
let $x_{n+1}, \ldots, x_{n+m}, w_{n+1}, \ldots, w_{n+m}$ be a quadrature rule for functions on the remaining portion of the curve $\partial \Omega \backslash \Gamma_{0}$, so that we have the approximation formulae

$$
\int_{\Gamma_{0}} f(x) d s(x) \approx \sum_{j=1}^{n} f\left(x_{j}\right) w_{j}
$$

and

$$
\int_{\partial \Omega \backslash \Gamma_{0}} f(x) d s(x) \approx \sum_{j=n+1}^{n+m} f\left(x_{j}\right) w_{j} .
$$

These quadratures lead to the system

$$
\sigma\left(x_{i}\right) \sqrt{w_{i}}+\sum_{j=1}^{n+m} K\left(x_{i}, y_{j}\right) \sigma\left(x_{j}\right) \sqrt{w_{i}} \sqrt{w_{j}}=2 g\left(x_{i}\right) \sqrt{w_{i}}, \quad i=1, \ldots, n+m,
$$

of $n+m$ linear equations in $n+m$ unknowns, which discretizes the integral equation (1.4).

The purpose of this algorithm is to replace the linear system (3.11) with a compressed system of $l+m$ linear equations in $l+m$ variables, where $l \ll n$. This is accomplished by constructing a quadrature rule $y_{1}, \ldots, y_{l}, v_{1}, \ldots, v_{l}$ such that the restrictions of solutions $\sigma$ of the equation (1.4) to $\Gamma_{0}$ are fully determined by their values at the quadrature nodes $y_{1}, \ldots, y_{l}$. This quadrature is used to discretize the self-interaction of $\Gamma_{0}$ as an $l \times l$ matrix and the interactions of $\Gamma_{0}$ with $\partial \Omega \backslash \Gamma_{0}$ through $l \times m$ and $m \times l$ matrices.

Step one: construction of a charge basis

The restricted integral equation (3.4) on $\Gamma_{0}$ is discretized using the quadrature rule with nodes $x_{1}, \ldots, x_{n}$ and weights $w_{1}, \ldots, w_{n}$. The resulting linear system is then solved for the functions (3.8), (3.9), and (3.10). The parameters $N_{1}, N_{2}$, and $N_{3}$, which control the number of right-hand sides, can be taken to be quite small because of the exponential convergence of the various approximations of the preceding section. For the experiments of this article, $N_{1}, N_{2}$, and $N_{3}$ were all taken to be 10. As discussed in Section 3.2, the resulting solutions, which we will denote by $\sigma_{1}, \sigma_{2}, \ldots, \sigma_{k}$, form a charge basis for the integral equation (1.4) over the region $\Gamma_{0}$.

Step two: formation of a generalized quadrature

In this step, a quadrature rule for integrals of the form

$$
\int_{\Gamma_{0}} K(x, y) \sigma_{j}(y) d s(y), \quad j=1, \ldots, k, \quad x \in \partial \Omega \backslash \Gamma_{0},
$$

is constructed. To accomplish this, we take advantage of the fact that the function $f: \Gamma_{0} \rightarrow \mathbb{R}$ defined by $f(y)=K(x, y)$ is piecewise smooth with a single discontinuity at the corner point of $\Gamma_{0}$ when $x \in \partial \Omega \backslash \Gamma_{0}$. This means that $f(y)$ can be approximated on $\Gamma_{0}$ by the images of piecewise polynomials on the intervals $[-\delta, 0]$ and $[0, \delta]$ under the parameterization $r:[-\delta, \delta] \rightarrow \Gamma_{0}$. In the first instance, an orthonormal basis $\phi_{1}, \ldots, \phi_{l}$ for the space spanned by the functions

$$
\sigma_{j}(x) p(x)+q(x), \quad j=1, \ldots, k,
$$

where $p(x)$ and $q(x)$ are images of piecewise polynomials of a fixed order, is formed. Then, the procedure described in Section 3.1 is applied to the functions $\phi_{1}, \ldots, \phi_{l}$ in order to generate the desired quadrature formula $y_{1}, \ldots, y_{l}, v_{1}, \ldots, v_{l}$. This quadrature rule is generally much smaller than the initial quadrature $x_{1}, \ldots, x_{n}, w_{1}, \ldots, w_{n}$. For the calculations of this article, we took the degree of the polynomials to be 9 .

Step three: construction of two transformation matrices 
Let $\Phi$ denote the inner product preserving mapping of the span of the $\phi_{1}, \ldots, \phi_{l}$ into $\mathbb{C}^{l}$ defined by

$$
\Phi\left(\sum_{i=1}^{l} \alpha_{i} \phi_{i}\right)=\left(\begin{array}{c}
\alpha_{1} \\
\alpha_{2} \\
\vdots \\
\alpha_{l}
\end{array}\right)
$$

and let $\Psi$ be the mapping of the span of $\phi_{1}, \ldots, \phi_{l}$ into $\mathbb{C}^{l}$ induced by the quadrature $y_{1}, \ldots, y_{l}, v_{1}, \ldots, v_{l}$ :

$$
\Psi(f)=\left(\begin{array}{c}
f\left(y_{1}\right) \sqrt{v_{1}} \\
f\left(y_{2}\right) \sqrt{v_{2}} \\
\vdots \\
f\left(y_{l}\right) \sqrt{v_{l}}
\end{array}\right) .
$$

The mapping $\Psi$ is not inner product preserving. Nonetheless, as described in [3], the matrices $\Psi \Phi^{-1}$ and $\Phi \Psi^{-1}$ exist and are well-conditioned. The matrix $\Psi \Phi^{-1}: \mathbb{C}^{l} \rightarrow \mathbb{C}^{l}$ takes the coefficient expansion of a function $f$ with respect to the basis $\phi_{1}, \ldots, \phi_{l}$ to its scaled values at the quadrature nodes (here, by scaled values, we mean the values of the function $f$ at the quadrature nodes $y_{1}, \ldots, y_{l}$ scaled by the square roots of the corresponding quadrature weights). Similarly, $\Phi \Psi^{-1}$, takes the scaled values of a function $f$ in the span of the $\phi_{1}, \ldots, \phi_{l}$ to its coefficient expansion with respect to $\phi_{1}, \ldots, \phi_{l}$.

In this step, we construct the $l \times l$ matrices $\Psi \Phi^{-1}$ and $\Phi \Psi^{-1}$ by first forming the matrix

$$
\Psi \Phi^{-1}=\left(\begin{array}{cccc}
\phi_{1}\left(y_{1}\right) \sqrt{v_{1}} & \phi_{2}\left(y_{1}\right) \sqrt{v_{1}} & \cdots & \phi_{l}\left(y_{1}\right) \sqrt{v_{1}} \\
\phi_{1}\left(y_{2}\right) \sqrt{v_{2}} & \phi_{2}\left(y_{2}\right) \sqrt{v_{2}} & \cdots & \phi_{l}\left(y_{2}\right) \sqrt{v_{2}} \\
\vdots & & \vdots & \\
\phi_{1}\left(y_{l}\right) \sqrt{v_{l}} & \phi_{2}\left(y_{l}\right) \sqrt{v_{l}} & \cdots & \phi_{l}\left(y_{l}\right) \sqrt{v_{l}}
\end{array}\right)
$$

and then inverting it to obtain $\Phi \Psi^{-1}$.

Step four: formation of the compressed linear system

In this final step, the system (3.11) of $n+m$ linear equations in $n+m$ unknowns is replaced by a compressed system of $l+m$ linear equations in $l+m$ unknowns of the form

$$
\left(\begin{array}{l}
s_{1} \\
s_{2}
\end{array}\right)+\left(\begin{array}{ll}
A_{11} & A_{12} \\
A_{21} & A_{22}
\end{array}\right)\left(\begin{array}{c}
s_{1} \\
s_{2}
\end{array}\right)=2\left(\begin{array}{c}
t_{1} \\
t_{2}
\end{array}\right)
$$

where:

$A_{11}$ is an $l \times l$ matrix discretizing the operator $T_{11}: L^{2}\left(\Gamma_{0}\right) \rightarrow L^{2}\left(\Gamma_{0}\right)$ defined by

$$
T_{11} \sigma(x)=\int_{\Gamma_{0}} K(x, y) \sigma(y) d s(y)
$$

$A_{12}$ is an $l \times m$ matrix discretizing the operator $T_{12}: L^{2}\left(\partial \Omega \backslash \Gamma_{0}\right) \rightarrow L^{2}\left(\Gamma_{0}\right)$ defined by

$$
T_{12} \sigma(x)=\int_{\partial \Omega \backslash \Gamma_{0}} K(x, y) \sigma(y) d s(y)
$$

$A_{21}$ is an $m \times l$ matrix discretizing the operator $T_{21}: L^{2}\left(\Gamma_{0}\right) \rightarrow L^{2}\left(\partial \Omega \backslash \Gamma_{0}\right)$ defined by

$$
T_{21} \sigma(x)=\int_{\Gamma_{0}} K(x, y) \sigma(y) d s(y) ; \text { and }
$$

$A_{22}$ is an $m \times m$ matrix discretizing the operator $T_{22}: L^{2}\left(\partial \Omega \backslash \Gamma_{0}\right) \rightarrow L^{2}\left(\partial \Omega \backslash \Gamma_{0}\right)$ defined by

$$
T_{22} \sigma(x)=\int_{\partial \Omega \backslash \Gamma_{0}} K(x, y) \sigma(y) d s(y)
$$


The $l \times l$ matrix $A_{11}$ is constructed by forming the product

$$
A_{11}=B \cdot\left(\Phi \Psi^{-1}\right)
$$

where $\Phi \Psi^{-1}$ is the transformation matrix from the preceding step and $B$ is the $l \times l$ matrix whose entries are

$$
B_{i j}=\sqrt{v_{i}} \int_{\Gamma_{0}} K\left(y_{i}, x\right) \phi_{j}(x) d s(x), \quad i=1, \ldots, l, \quad j=1, \ldots, l .
$$

Note that the entries of $B$ can be calculated with the initial quadrature rule $x_{1}, \ldots, x_{n}, w_{1}, \ldots, w_{n}$ for the corner region $\Gamma_{0}$. That $A_{11}$ approximates the operator $T_{11}$ can be seen easily. The matrix $\Phi \Psi^{-1}$ maps the scaled values of functions in the span of the basis $\phi_{1}, \ldots, \phi_{l}$ to their coefficient expansions and the matrix $B$ takes coefficient expansions to the scaled values of their images under the operator $T_{11}$.

The matrix $A_{22}$, which corresponds to the self-interaction of the curve segment $\partial \Omega \backslash \Gamma_{0}$, is formed in the usual fashion; that is, $A_{22}$ is the $m \times m$ matrix with entries

$$
\left(A_{22}\right)_{i j}=K\left(x_{n+i}, x_{n+j}\right) \sqrt{w_{n+i}} \sqrt{w_{n+j}}, \quad i=1, \ldots, m, \quad j=1, \ldots m .
$$

The matrices $A_{12}$ and $A_{21}$, which approximate the interactions of $\Gamma_{0}$ with $\partial \Omega \backslash \Gamma_{0}$, are discretized through the quadrature $y_{1}, \ldots y_{l}, v_{1}, \ldots, v_{l}$. Specifically, $A_{12}$ is taken to be the $l \times m$ matrix with entries

$$
\left(A_{12}\right)_{i j}=K\left(y_{i}, x_{n+j}\right) \sqrt{w_{n+j}} \sqrt{v_{i}}, \quad i=1, \ldots, l, \quad j=1, \ldots, m,
$$

and $A_{21}$ is taken to be the $m \times l$ matrix with entries

$$
\left(A_{21}\right)_{i j}=K\left(x_{n+i}, y_{j}\right) \sqrt{w_{n+i}} \sqrt{v_{j}}, \quad i=1, \ldots, m, \quad j=1, \ldots, l .
$$

Finally, we form the right-hand side of the system (3.13) by setting

$$
t_{1}=\left(\begin{array}{c}
g\left(y_{1}\right) \sqrt{v_{1}} \\
g\left(y_{2}\right) \sqrt{v_{2}} \\
\vdots \\
g\left(y_{l}\right) \sqrt{v_{l}}
\end{array}\right) \quad \text { and } \quad t_{2}=\left(\begin{array}{c}
g\left(x_{n+1}\right) \sqrt{w_{n+1}} \\
g\left(x_{n+2}\right) \sqrt{w_{n+2}} \\
\vdots \\
g\left(x_{n+m}\right) \sqrt{w_{n+m}}
\end{array}\right) \text {. }
$$

The unknowns in the compressed linear system are the vectors

$$
s_{1}=\left(\begin{array}{c}
\sigma\left(y_{1}\right) \sqrt{v_{1}} \\
\sigma\left(y_{2}\right) \sqrt{v_{2}} \\
\vdots \\
\sigma\left(y_{l}\right) \sqrt{v_{l}}
\end{array}\right) \quad \text { and } \quad s_{2}=\left(\begin{array}{c}
\sigma\left(x_{n+1}\right) \sqrt{w_{n+1}} \\
\sigma\left(x_{n+2}\right) \sqrt{w_{n+2}} \\
\vdots \\
\sigma\left(x_{n+m}\right) \sqrt{w_{n+m}}
\end{array}\right) \text {. }
$$

Note that the scaled values of a charge distribution $\sigma$ at the quadrature nodes $y_{1}, \ldots, y_{l}$ are sufficient to fully specify the restriction of the $\sigma$ to $\Gamma_{0}$. Indeed, a coefficient expansion for the restriction in terms of the basis $\phi_{1}, \ldots, \phi_{l}$ can be computed by applying the transformation matrix $\Phi \Psi^{-1}$ constructed in the preceding section to the vector $s_{1}$.

Remark 3.2. The procedure of this section applies to many boundary integral operators, not just those associated with Laplace's equation. See, for instance, [3] for a discussion of the application of these ideas to the boundary integral operators arising from the Helmholtz equation. Moreover, it can extended to more general partial differential equations, including certain classes of equations with nonconstant coefficients. This generalization will be reported at a later date.

Remark 3.3. The algorithm of [2] produces smaller compressed systems than the procedure of this section, but it does so at the cost of greater complexity. Rather than using a quadrature for the functions (3.12) to discretize the interactions of the curve segment $\Gamma_{0}$, it uses a quadrature for the smaller collection of functions

$$
\sigma_{j}(x), \quad j=1, \ldots, k
$$


The advantage of this approach is, of course, that the resulting compressed systems of linear equations are smaller. The disadvantage is that interpolatory quadratures must used to evaluate the integrals

$$
\int_{\Gamma_{0}} K(x, y) \sigma_{j}(y) d s(y)
$$

when $x \in \partial \Omega \backslash \Gamma_{0}$. Depending the solver applied to the compressed linear systems, this approach can actually result in a less efficient algorithm.

\section{NumERICAL EXPERIMENTS}

This section describes several numerical experiments performed to assess the accuracy and stability of inner product preserving Nyström discretizations of (1.4) and to compare such discretizations to those obtained via the standard Nyström approach.

Although the computations described here could be accelerated greatly through the use of fast multipole methods and fast direct solvers, for the sake of simplicity and in the interests of reproducibility the experiments were conducted using standard LAPACK routines. In particular, singular value decompositions, which were used to solve all linear systems save for those related to the compression scheme of Section 3, were constructed using the routine DGESDD. Linear systems arising from the compression scheme were solved using the DGESV routine.

Note also that the accuracy of solutions was given priority over the efficiency of discretizations. Substantially more efficient discretizations can be obtained in some cases by sacrificing a few digits of precision.

All experiments were performed on a PC equipped with a $2.54 \mathrm{GHz}$ Intel Core 2 Duo processor and 8GB of RAM. Code for the experiments was written in Fortran 77 and compiled with the Intel Fortran Compiler, version 11.1.

4.1. Graded meshes. Before beginning the description of the experiments proper, we need to introduce the terminology which will be used throughout this section to describe quadratures for the discretization of integral operators. Let $\partial \Omega$ be a boundary curve parameterized by the mapping $r:[a, b] \rightarrow \partial \Omega \subset R^{2}$. A quadrature formula on the interval $[a, b]$ can be mapped into $\partial \Omega$ via the parameterization $r$. More specifically, the quadrature rule $x_{1}, \ldots, x_{n}, w_{1}, \ldots, w_{n}$ on $[a, b]$ induces the approximation formula

$$
\int_{\Gamma} f(x) d s(x) \approx \sum_{j=1}^{n} f\left(x_{j}\right)\left|r^{\prime}\left(x_{j}\right)\right| w_{j} .
$$

In what follows, we will implicitly identify the parameterization domain $[a, b]$ and the boundary curve $\partial \Omega$ through the use of this mapping. That is, we will describe quadratures on the curve $\partial \Omega$ by describing their preimage in the parameterization domain $[a, b]$ under this mapping.

To that end, we shall call the piecewise Legendre quadrature of order $m$ on the intervals

$$
\left[-2^{-j+1},-2^{-j}\right], \quad\left[2^{-j}, 2^{-j+1}\right], \quad j=1, \ldots, N,
$$

a simply-graded mesh ${ }^{1}$ of order $m$ on $[-1,1]$ with cutoff $2^{-N}$. We call the image of such a quadrature under the substitution

$$
u=x^{k},
$$

where $k$ is a positive odd integer, a graded mesh on $[-1,1]$ of order $m$, exponent $k$, and cutoff $2^{-k N}$. Note that the quadrature formula associated with a graded mesh on $[-1,1]$ is

$$
\int_{-1}^{1} f(x) d x \approx \sum_{j=1}^{l} f\left(x_{j}^{k}\right) k x_{j}^{k-1} w_{j},
$$

where $x_{1}, \ldots, x_{l}$ and $w_{1}, \ldots, w_{l}$ denote the nodes and weights of the original piecewise Legendre quadrature.

In order to form a quadrature for a boundary curve with corner points parameterized over $[a, b]$ we will use a combination of graded meshes and piecewise Legendre quadratures. For each corner

\footnotetext{
${ }^{1}$ The term simply-graded is borrowed from [7].
} 
point $r\left(t_{j}\right)$ on the boundary curve, we form a graded mesh on $[-1,1]$ and map it affinely into a neighborhood $\left[t_{j}-\delta, t_{j}+\delta\right]$ of $t_{j}$. A quadrature on the remaining portion of the interval $[a, b]$ is then formed using a piecewise Legendre rule. In many of the following experiments, we will describe graded meshes using these 4 parameters: the order $m$ of the piecewise Legendre quadrature used to form the graded mesh, the radius $\delta$, the cutoff $\epsilon_{\text {cut }}$ for the mesh, and the exponent $k$.

4.2. A family of domains with a single outward-pointing corner. For $0<\theta<\pi$, let $\Omega_{\theta}$ denote the domain bounded by the simply-connected curve parameterized over the torus $[-\pi, \pi]$ by

$$
\begin{aligned}
& x_{\theta}(t)= \begin{cases}-2 \sin (t / 2) & -\pi<t<0, \\
2 \sin (t / 2) & 0<t<\pi\end{cases} \\
& y_{\theta}(t)=-\tan (\theta / 2) \sin (t) .
\end{aligned}
$$

The parameterization $\left(x_{\theta}(t), y_{\theta}(t)\right)$ has counter-clockwise orientation and maps $t=0$ to a corner point with interior angle $\theta$ at the origin; Figure 2 depicts $\Omega_{\pi / 2}$. We will refer to the $\Omega_{\theta}$ as "snowcone" domains.

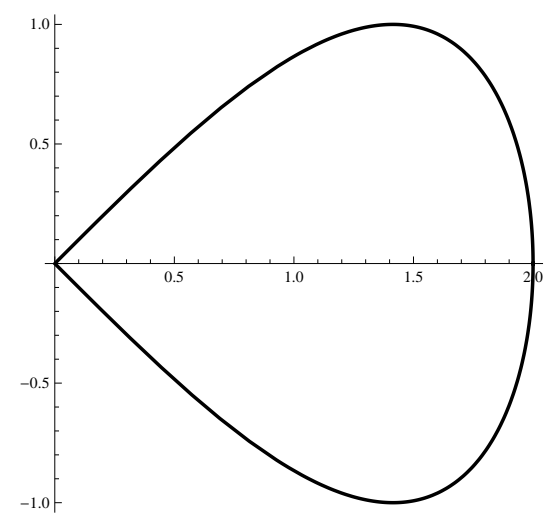

Figure 2: The domain $\Omega_{\pi / 2}$.
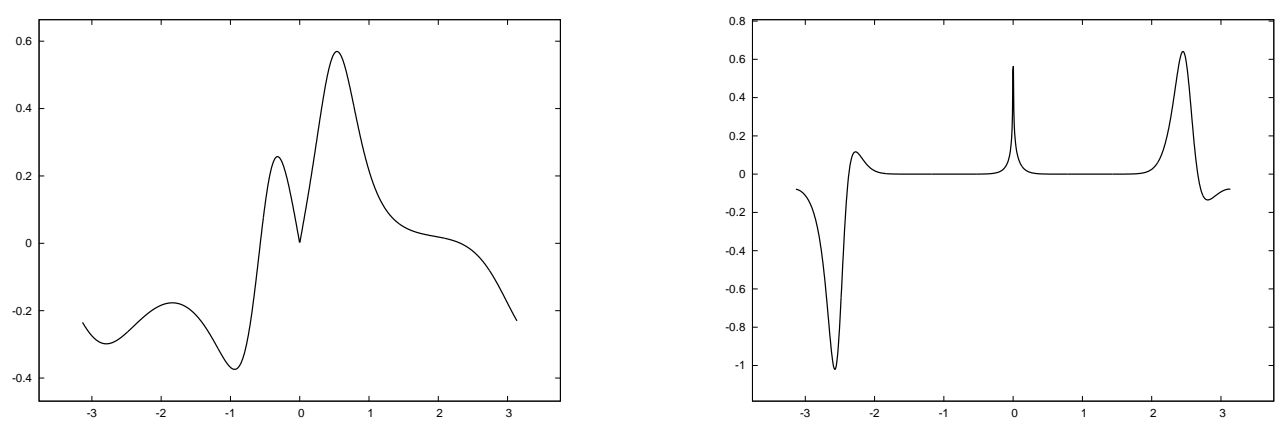

Figure 3: The single-layer potentials on $\partial \Omega_{\pi / 2}$ (left) and $\partial \Psi_{3 \pi / 2}$ (right) which generate the harmonic function (4.1). Note that the function on the right has an unbounded singularity at 0 which has been truncated.

Several discretizations of the operator

$$
T \sigma(x)=\sigma(x)+\int_{\partial \Omega_{\pi / 2}}\left(\frac{\partial}{\partial \nu_{x}} \log |x-y|\right) \sigma(y) d s(y)
$$

were formed using various graded mesh quadratures. In each case, the mesh parameters $m$ and $\delta$ were 30 and 1, respectively, and an 180-point piecewise Legendre quadrature was used to discretize the operator over the smooth portions of the curve - that is, the regions parameterized over the 
intervals $[-\pi,-1]$ and $[1, \pi]$. For each graded mesh quadrature considered, both the inner product preserving discretization (2.2) and the usual Nyström discretization (2.3) were formed. Moreover, each inner product preserving discretization was used to compute a solution to an exterior Neumann problem on the domain $\Omega_{\pi / 2}$. The boundary data was taken to be the restriction to $\partial \Omega_{\pi / 2}$ of the normal derivative of the function

$$
u(x)=\log \left|x-z_{0}\right|-\log \left|x-z_{1}\right|,
$$

where $z_{0}=(1.0,0.0)$ and $z_{1}=(1.1,0.1)$. Note that

$$
u(x)=O(1 /|x|) \text { as }|x| \rightarrow \infty .
$$

For each obtained solution, the error in the representation (1.3) was measured at 300 points on the circle $C$ of radius 3 centered at the origin. The results appear in Table 1; the quantities reported are as follows:

- $\epsilon_{\text {cut }}$ refers to the cutoff for the graded mesh;

- $k$ is the exponent of the graded mesh;

- $n$ is the dimension of the systems of linear equations;

- $\kappa$ is the condition number of the inner product preserving discretization;

- $E$ is the largest absolute error observed while testing the representation (1.3) on the circle $C$;

- $\kappa_{N}$ is the condition number of the standard Nyström discretization.

\begin{tabular}{llllll}
\hline$\epsilon_{\text {cut }}$ & $k$ & $n$ & $\kappa$ & $E$ & $\kappa_{N}$ \\
\hline $1.0 \times 10^{-7}$ & 1 & 1620 & $5.55 \times 10^{+0}$ & $3.66 \times 10^{-15}$ & $5.82 \times 10^{+4}$ \\
$1.0 \times 10^{-15}$ & 1 & 3180 & $6.44 \times 10^{+0}$ & $4.44 \times 10^{-16}$ & $4.59 \times 10^{+7}$ \\
$1.0 \times 10^{-7}$ & 3 & 660 & $5.55 \times 10^{+0}$ & $3.11 \times 10^{-15}$ & $5.31 \times 10^{+4}$ \\
$1.0 \times 10^{-15}$ & 3 & 1200 & $6.44 \times 10^{+0}$ & $1.11 \times 10^{-14}$ & $5.01 \times 10^{+7}$ \\
$1.0 \times 10^{-30}$ & 3 & 2200 & $6.82 \times 10^{+0}$ & $3.33 \times 10^{-16}$ & $1.11 \times 10^{+13}$ \\
$1.0 \times 10^{-7}$ & 5 & 480 & $5.55 \times 10^{+0}$ & $2.55 \times 10^{-15}$ & $5.79 \times 10^{+4}$ \\
$1.0 \times 10^{-15}$ & 5 & 780 & $6.44 \times 10^{+0}$ & $6.66 \times 10^{-16}$ & $4.21 \times 10^{+7}$ \\
$1.0 \times 10^{-30}$ & 5 & 1380 & $6.82 \times 10^{+0}$ & $1.55 \times 10^{-15}$ & $8.23 \times 10^{+12}$ \\
$1.0 \times 10^{-7}$ & 7 & 420 & $5.55 \times 10^{+0}$ & $3.00 \times 10^{-15}$ & $7.88 \times 10^{+4}$ \\
$1.0 \times 10^{-15}$ & 7 & 660 & $6.44 \times 10^{+0}$ & $7.77 \times 10^{-16}$ & $7.95 \times 10^{+7}$ \\
$1.0 \times 10^{-30}$ & 7 & 1080 & $6.82 \times 10^{+0}$ & $1.00 \times 10^{-14}$ & $1.33 \times 10^{+13}$ \\
\hline
\end{tabular}

Table 1: Numerical results for the snowcone domain $\Omega_{\pi / 2}$.

Figure 3 shows the single layer potential on $\partial \Omega_{\pi / 2}$ which gives rise to the harmonic function (4.1) in $\Omega_{\pi_{2}}^{c}$. It is clear from Table 1 that inner product preserving discretization leads to well-conditioned systems while the standard Nyström approach results in numerical instability. It is also clear that high accuracy can be achieved for this problem.

We also solved exterior Neumann problems on a collection of domains $\Omega_{\theta}$ with angles $\theta$ close to 0 and $\pi$ via the integral equations

$$
\sigma(x)+\int_{\partial \Omega_{\theta}}\left(\frac{\partial}{\partial \nu_{x}} \log |x-y|\right) \sigma(y) d s(y)=2 g(x), \quad x \in \partial \Omega_{\theta} .
$$

For these experiments, inner product preserving discretizations were used and the boundary data $g$ was taken to be the normal derivative of the potential

$$
\log \left|x-z_{2}\right|-\log \left|x-z_{3}\right|
$$

where $z_{2}=(1,0)$ and $z_{3}=(1.1,0)$. These locations were chosen because $z_{2}$ and $z_{3}$ are contained in the domains $\Omega_{\theta}$ for all values of $\theta$. Table 2 reports the results of these experiments. There, $\theta$ 
refers to the angle of the corner, $n$ is the number of quadrature nodes used to discretize the integral equation, $\kappa$ is the condition number of the system of linear equations, and $E$ is the largest absolute error observed while testing the representation (1.3) at a collection of 300 points sampled randomly in the box with corners $(-5,-1)$ and $(-4,1)$.

\begin{tabular}{llll}
\hline$\theta$ & $n$ & $\kappa$ & $E$ \\
\hline$\pi / 10$ & 960 & $3.28 \times 10^{+1}$ & $6.66 \times 10^{-16}$ \\
$\pi / 20$ & 1080 & $6.90 \times 10^{+1}$ & $8.88 \times 10^{-16}$ \\
$\pi / 100$ & 1800 & $4.70 \times 10^{+2}$ & $5.55 \times 10^{-15}$ \\
$\pi / 1000$ & 1980 & $5.09 \times 10^{+4}$ & $1.25 \times 10^{-12}$ \\
$\pi-\pi / 10$ & 1080 & $1.06 \times 10^{+1}$ & $8.88 \times 10^{-16}$ \\
$\pi-\pi / 20$ & 1140 & $2.09 \times 10^{+1}$ & $2.00 \times 10^{-15}$ \\
$\pi-\pi / 100$ & 1440 & $1.03 \times 10^{+2}$ & $1.02 \times 10^{-14}$ \\
$\pi-\pi / 1000$ & 3480 & $2.64 \times 10^{+4}$ & $5.12 \times 10^{-11}$ \\
\hline
\end{tabular}

Table 2: Results for nearly degenerate snowcone domains.

It is expected that the conditioning of the linear systems discretizing (4.2) will deteriorate as $\theta$ goes to 0 and $\pi$. This phenomenon is not related to the corner singularity. It is instead a consequence of the fact that the domains $\Omega_{\theta}$ become elongated as $\theta$ goes to 0 and $\pi$. The same behavior can be observed in potential theoretic operators on eccentric ellipses. Let $D$ be the integral operator

$$
D f(x)=\frac{1}{\pi} \int_{\partial E}\left(\frac{\partial}{\partial \nu_{y}} \log |x-y|\right) f(y) d s(y),
$$

where $\partial E$ is the counter-clockwise oriented boundary of the ellipse

$$
\left(\frac{x}{a}\right)^{2}+\left(\frac{y}{b}\right)^{2}<1
$$

The operator $D$ arises in the solution of interior Dirichlet problems for Laplace's equation. In particular, $I+D$ plays the same in role for Dirichlet problems that the operator $I+N$ does for Neumann problems. We can assume that $a>b>0$. Then the mapping $[-\pi, \pi] \rightarrow \mathbb{R}^{2}$ defined by

$$
\begin{aligned}
& x(t)=a \cos (t) \\
& y(t)=b \sin (t)
\end{aligned}
$$

is a counter-clockwise oriented parameterization for $\partial E$. It allows us to rewrite $D$ as

$$
D f(t)=\frac{1}{\pi} \int_{-\pi}^{\pi} \frac{a b}{a^{2}+b^{2}+\left(b^{2}-a^{2}\right) \cos (s+t)} f(s) d s .
$$

The spectrum of $D$ can be easily computed since it is a convolution operator. Its eigenvalues $\left\{\lambda_{n}\right\}_{n=-\infty}^{\infty}$ are

$$
\lambda_{n}=\left(\frac{a-b}{a+b}\right)^{n}, n=0, \ldots, \infty,
$$

and

$$
\lambda_{-n}=-\left(\frac{a-b}{a+b}\right)^{n}, \quad n=1, \ldots, \infty .
$$

It follows that the spectrum of the operator $I+D$ is the set

$$
\sigma(I+D)=\{2\} \cup\left\{1 \pm\left(\frac{a-b}{a+b}\right)^{n} \mid n=1, \ldots, \infty\right\} .
$$

From this, we see that the $L^{2}(\partial E)$ condition number of the operator $I+D$ is

$$
\|I+D\|_{2}\left\|(I+D)^{-1}\right\|_{2}=1+\frac{a}{b}
$$


This condition number increases as the ratio of $a$ to $b$ becomes large; i.e., as the ellipse $E$ becomes more eccentric. A similar, but slightly more complicated, analysis applies to the operator $I+N$ associated with the Neumann problem for Laplace's equation.

4.3. A family of domains with a single inward-pointing corner. In this section, we describe several numerical experiments involving a family of "boomerang" domains which have a single inward-pointing corner. For $\pi<\theta<2 \pi$, let $\Psi_{\theta}$ be the simply-connected domain whose boundary is parameterized over $[\pi, \pi]$ by

$$
\begin{aligned}
& x_{\theta}(t)= \begin{cases}2 \sin (3 t / 2) & -\pi \leq t<0, \\
-2 \sin (3 t / 2) & 0<t \leq \pi\end{cases} \\
& y_{\theta}(t)=\tan (\theta / 2) \sin (t)
\end{aligned}
$$

The domain $\Psi_{\theta}$ has a single corner point with interior angle $\theta$ at the origin; see Figure 4, which depicts $\Psi_{3 \pi / 2}$.

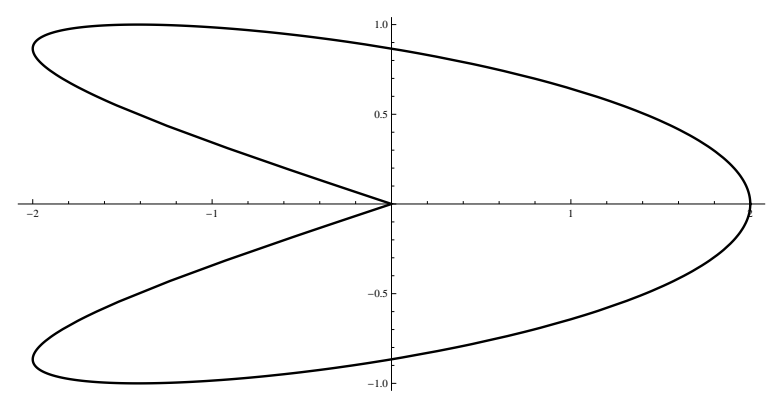

Figure 4: The domain $\Psi_{3 \pi / 2}$.

\begin{tabular}{llllll}
\hline$\epsilon_{\text {cut }}$ & $k$ & $n$ & $\kappa$ & $E$ & $\kappa_{N}$ \\
\hline $1.0 \times 10^{-15}$ & 1 & 3600 & $3.41 \times 10^{+1}$ & $1.18 \times 10^{-10}$ & $3.27 \times 10^{9}$ \\
$1.0 \times 10^{-15}$ & 3 & 1620 & $3.37 \times 10^{+1}$ & $2.54 \times 10^{-10}$ & $1.59 \times 10^{9}$ \\
$1.0 \times 10^{-25}$ & 3 & 2280 & $3.91 \times 10^{+1}$ & $6.66 \times 10^{-16}$ & $6.13 \times 10^{13}$ \\
$1.0 \times 10^{-30}$ & 3 & 2640 & $4.07 \times 10^{+1}$ & $4.44 \times 10^{-16}$ & $1.41 \times 10^{16}$ \\
$1.0 \times 10^{-30}$ & 5 & 1800 & $4.05 \times 10^{+1}$ & $3.72 \times 10^{-12}$ & $5.22 \times 10^{15}$ \\
\hline
\end{tabular}

Table 3: Numerical results for the boomerang domain $\Psi_{3 \pi / 2}$.

We formed several discretizations of the integral equation

$$
\sigma(x)+\int_{\partial \Psi_{3 \pi / 2}}\left(\frac{\partial}{\partial \nu_{x}} \log |x-y|\right) \sigma(y) d s(y)=2 g(x), \quad x \in \partial \Psi_{3 \pi / 2},
$$

with $g(x)$ taken to be the normal derivative of the function defined by (4.1). A number of different graded meshes were used and both inner product preserving and standard Nyström discretizations were formed for each mesh. In all cases, the parameters $\delta$ and $m$ were taken to be $1 / 2$ and 30 , respectively, and the integral equation was discretized over smooth portions of the curve using piecewise Legendre quadratures. The results appear in Table 3; the quantities reported are the same as those in Table 1 of the preceding section. The charge distribution $\sigma$ which gives rise to the harmonic function (4.1) in $\Psi_{3 \pi / 2}^{c}$ is shown in Figure 3. Note that $\sigma$ has an unbounded singularity at 0 . 


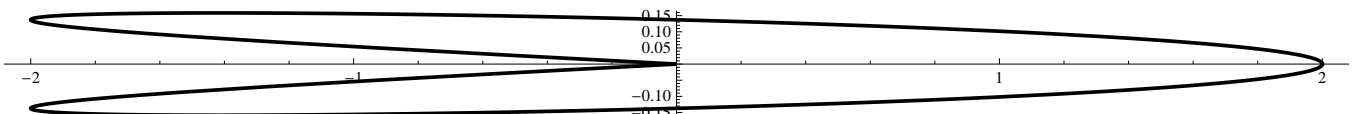

Figure 5: The domain $\Psi_{2 \pi-\pi / 10}$.

We also solved exterior Neumann problems on a collection of domains $\Psi_{\theta}$ with angles $\theta$ close to $\pi$ and $2 \pi$ via inner product preserving discretizations of the integral equations

$$
\sigma(x)+\int_{\partial \Psi_{\theta}}\left(\frac{\partial}{\partial \nu_{x}} \log |x-y|\right) \sigma(y) d s(y)=2 g(x), \quad x \in \partial \Psi_{\theta} .
$$

For each problem, the boundary data was taken to be the normal derivative of the function (4.3). Table 4 presents the results of these experiments; the quantities reported are the same as those in Table 2. Note that the resulting charge distributions exhibit unbounded singularities in the case of angles near $\pi$ but not in the case of angles near $2 \pi$; see, for instance, Figure 6 , which shows the single-layer potentials on $\partial \Psi_{2 \pi-\pi / 10}$ and $\partial \Psi_{\pi+\pi / 10}$ which generate the harmonic function (4.3).
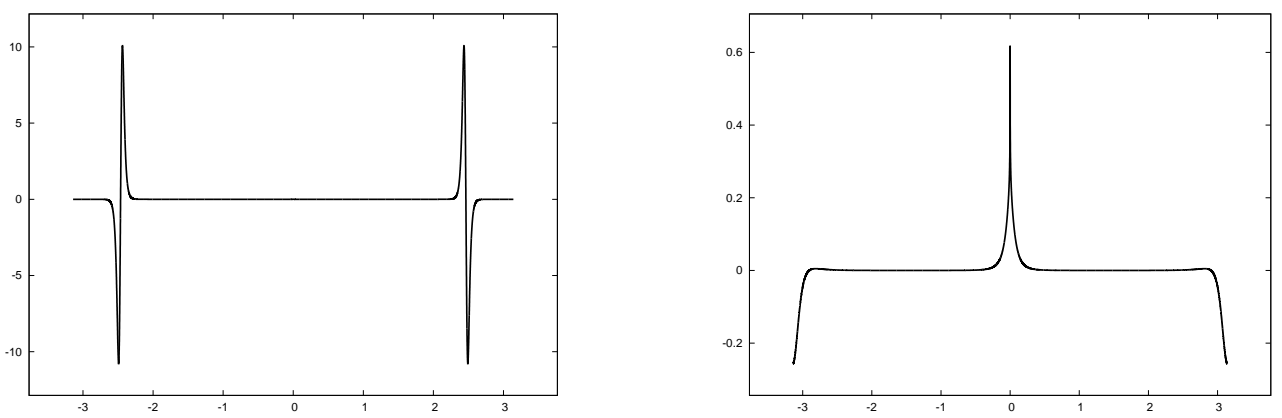

Figure 6: The single-layer potentials on $\partial \Psi_{2 \pi-\pi / 10}$ (left) and $\partial \Psi_{\pi+\pi / 10}$ (right) which generate the function (4.3).

Nonetheless, much more difficulty was encountered in the solution of the Neumann problems when $\theta$ was close to $2 \pi$ than when $\theta$ was close to $\pi$. These difficulties do not arise because of the corner singularities, but because of the elongated segments of the boundary contours. Figure 5 shows the domain $\Psi_{2 \pi-\pi / 10}$, whose boundary features two elongated regions.

\begin{tabular}{llll}
\hline$\theta$ & $n$ & $\kappa$ & $E$ \\
\hline$\pi+\pi / 10$ & 1680 & $7.59 \times 10^{+0}$ & $1.11 \times 10^{-14}$ \\
$\pi+\pi / 20$ & 1800 & $1.22 \times 10^{+1}$ & $7.55 \times 10^{-15}$ \\
$\pi+\pi / 100$ & 1860 & $5.53 \times 10^{+1}$ & $5.40 \times 10^{-14}$ \\
$\pi+\pi / 1000$ & 4080 & $5.56 \times 10^{+2}$ & $3.70 \times 10^{-12}$ \\
$2 \pi-\pi / 10$ & 4440 & $2.09 \times 10^{+3}$ & $2.46 \times 10^{-14}$ \\
$2 \pi-\pi / 20$ & 2280 & $1.06 \times 10^{+4}$ & $4.33 \times 10^{-14}$ \\
$2 \pi-\pi / 100$ & 3808 & $2.85 \times 10^{+5}$ & $6.84 \times 10^{-14}$
\end{tabular}

Table 4: Results for nearly degenerate boomerang domains.

4.4. A domain with 8 corner points. In this experiment, we solved an exterior Neumann problem on the simply-connected "inkblot" domain $\Omega_{\text {ink }}$ shown in Figure 7 . The boundary of this domain has 8 corner points, 4 inward-pointing and 4 outward-pointing, and is parameterized by the polar equation

$$
r(\theta)=4+2|\cos (4 \theta)| \sin (4 \theta), \quad 0 \leq \theta \leq 2 \pi .
$$


The boundary data $g$ was taken to be the normal derivative of a potential generated by two charges of equal magnitude and opposite signs which were randomly placed in the interior of $\Omega_{\mathrm{ink}}$.

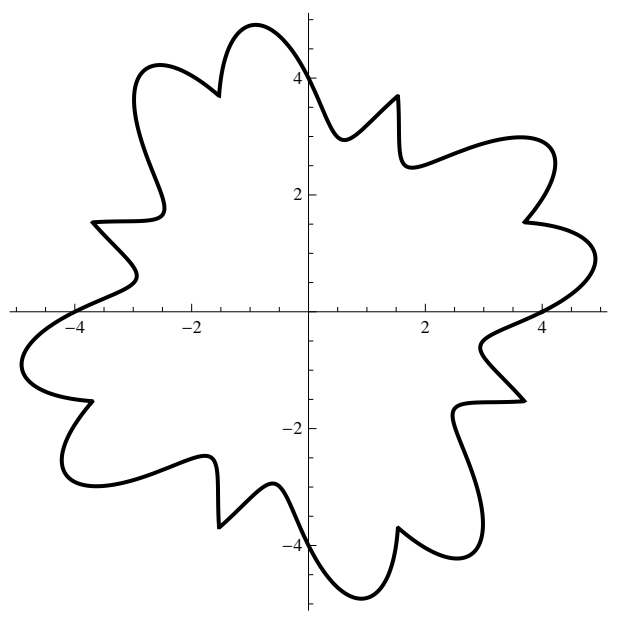

Figure 7: The domain $\Omega_{\text {ink }}$ of Section 4.4.

\begin{tabular}{llllllll}
\hline & $n$ & $n_{\text {corner }}$ & $n_{\text {smooth }}$ & $\kappa$ & $E$ & $T_{\text {SVD }}$ & $T_{\text {compress }}$ \\
\hline Uncompressed system & 8700 & 1020 & 540 & $1.95 \times 10^{+1}$ & $9.27 \times 10^{-15}$ & 1112.5 & - \\
Compressed system & 1004 & 58 & 540 & $5.41 \times 10^{+1}$ & $5.55 \times 10^{-15}$ & 1.34 & 3.26 \\
\hline
\end{tabular}

Table 5: Numerical results for the domain $\Omega_{\text {ink }}$ of Section 4.4. Entries marked with a dash are not applicable.

In order to obtain the solution of this Neumann problem, an inner product preserving discretization of the integral equation

$$
\sigma(x)+\int_{\partial \Omega_{\mathrm{ink}}}\left(\frac{\partial}{\partial \nu_{x}} \log |x-y|\right) \sigma(y) d s(y)=2 g(x), \quad x \in \partial \Omega_{\mathrm{ink}},
$$

was formed and a singular value decomposition of the matrix associated with this linear system was computed and used to solve the linear system. The resulting charge distribution is singular at each corner point of $\partial \Omega_{\text {ink }}$; the singularities occurring at inward-pointing corner points are unbounded while those at outward-pointing corner points are bounded. The compression scheme of Section 3 was also applied to this discretization in order to form a compressed system of equations. Table 5 presents the results; the quantities reported are as follows:

- $n$ is the dimension of the system of linear equations;

- $n_{\text {corner }}$ is the average number of nodes used to discretize corner regions;

- $n_{\text {smooth }}$ is the number of quadrature nodes used to discretize the smooth portions of the curve;

- $\kappa$ is the condition number of system;

- $E$ is the largest absolute error in the representation (1.3) occurring on the circle of radius 6 centered at the origin;

- $T_{\mathrm{SVD}}$ is the wall-clock time, in seconds, which was required to compute the singular value decomposition of the matrix;

- $T_{\text {compress }}$ is the wall-clock time, in seconds, required to execute the corner compression procedure. 
4.5. A polygonal domain with 38 corner points. In this final experiment, an inner product preserving discretization of the the integral equation

$$
\sigma(x)+\int_{\partial \Omega_{\text {star }}}\left(\frac{\partial}{\partial \nu_{x}} \log |x-y|\right) \sigma(y) d s(y)=2 g(x), \quad x \in \partial \Omega_{\text {star }},
$$

where $\Omega_{\text {star }}$ is the simply-connected "starburst" domain shown in Figure 8, was formed. The boundary of $\Omega_{\text {star }}$ is a polygon with 38 vertices. The procedure of Section 3, which is absolutely essential in this case, was applied to that discretization in order to form a compressed system of linear equations. The compressed linear system was used to solve the exterior Neumann problem on $\Omega_{\text {star }}$ for boundary data which was the normal derivative of a potential generated by two charges of equal magnitude and opposite sign placed randomly in the domain. Table 6 describes the results; the quantities reported are the same as those of Table 5 in Section 4.4.

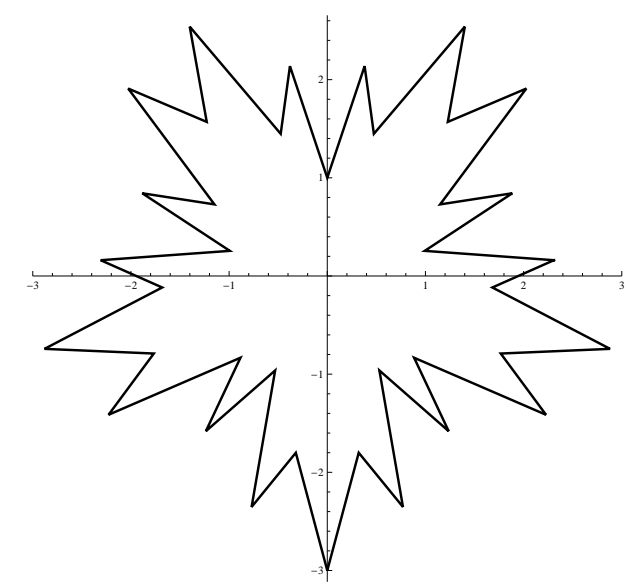

Figure 8: The starburst domain $\Omega_{\text {star }}$.

\begin{tabular}{llllllll}
\hline & $n$ & $n_{\text {corner }}$ & $n_{\text {smooth }}$ & $\kappa$ & $E$ & $T_{\text {SVD }}$ & $T_{\text {compress }}$ \\
\hline Uncompressed system & 70740 & 1800 & 2340 & $*$ & $*$ & $*$ & - \\
Compressed system & 5198 & 75.21 & 2340 & $1.40 \times 10^{+2}$ & $1.45 \times 10^{-15}$ & 223.2 & 84.2 \\
\hline
\end{tabular}

Table 6: Numerical results for the domain $\Omega_{\text {star }}$. Entries marked with a dash are not applicable, while those marked with an asterisk could not be calculated due to computational limitations.

\section{Conclusions}

There is an extant problem regarding the solution of boundary integral equations on planar domains with corners, but it is not the stability or accuracy of graded mesh discretization. Rather, the outstanding issue is the compression of the large systems of linear equations which result from the discretization of boundary integral equations on such domains. Of particular interest are schemes, like those presented in [7] and [3, 2], which do not require a priori analytic information about the behavior of solutions. This property allows them to be applied to the many problems which can be formulated as boundary integral equations with little modification. Moreover, it is almost a prerequisite for schemes in the three-dimensional environment, where estimates of the behavior of solutions are difficult to obtain. Indeed, one of the principal advantages of the approach of Section 3 is that it admits generalization to three-dimensional domains with singularities, a generalization which will be reported by the author at a later date. 
ON THE NYSTRÖM DISCRETIZATION OF INTEGRAL EQUATIONS ON PLANAR CURVES WITH CORNERS1

\section{AcKnowledgements}

The author is grateful to Vladimir Rokhlin for the benefit of his extensive knowledge of the numerical solution of boundary integral equations. This work was supported by the Office of Naval Research, under contract N00014-09-1-0318.

\section{REFERENCES}

[1] J. Bremer, Z. Gimbutas, and V. Rokhlin, A nonlinear optimization procedure for generalized Gaussian quadratures, SIAM Journal of Scientific Computing, 32 (2010), pp. 1761-1788.

[2] J. BRemer AND V. RoKhlin, Efficient discretization of Laplace boundary integral equations on polygonal domains, Journal of Computational Physics, 229 (2010), pp. 2507-2525.

[3] J. Bremer, V. Rokhlin, AND I. SAmmis, Universal quadratures for boundary integral equations on twodimensional domains with corners, Journal of Computational Physics, to appear (2010).

[4] O. P. Bruno, J. S. Ovall, And C. Turc, A high-order integral algorithm for highly singular PDE solutions in Lipschitz domains, Computing, 84 (2009), pp. 149-181.

[5] R. Coifman And Y. Meyer, Wavelets: Calderón-Zygmund and Multilinear Operators, Cambridge University Press, 1997.

[6] M. Gu AND S. EISEnStat, Efficient algorithms for computing a strong rank-revealing QR factorization, SIAM Journal of Scientific Computing, 17 (1996), pp. 848-869.

[7] J. Helsing AND R. OJala, Corner singularities for elliptic problems: integral equations, graded meshes, quadrature, and compressed inverse preconditioning, Journal of Computational Physics, 227 (2008), pp. 8820-8840.

[8] R. Kress, Integral Equations, Springer-Verlag, New York, 1999. 